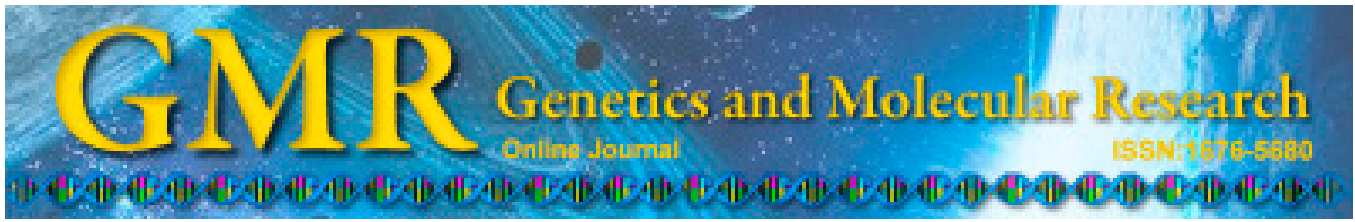

\title{
Correlation between ERCC1 expression and concurrent chemotherapy and radiotherapy in patients with locally advanced nasopharyngeal cancer
}

\author{
R. Liang, Y. Lin, Z.H. Liu, X.L. Liao, C.L. Yuan, S.N. Liao and Y.Q. Li \\ Department of First Chemotherapy, \\ Affiliated Cancer Hospital of Guangxi Medical University, Nanning, China \\ Corresponding author: Y.Q. Li \\ E-mail: lingrong_1r@163.com
}

Genet. Mol. Res. 14 (2): 5804-5811 (2015)

Received November 6, 2014

Accepted February 9, 2015

Published May 29, 2015

DOI http://dx.doi.org/10.4238/2015.May.29.12

\begin{abstract}
In this study, the expression of DNA excision repair cross-complementing gene 1 (ERCC1) in local advanced nasopharyngeal carcinoma has been correlated with the efficacy of concurrent chemoradiotherapy. A total of 76 patients diagnosed with undifferentiated nasopharyngeal carcinoma diagnosed by nasopharyngeal biopsy and undergoing single-agent cisplatin chemotherapy $\left(80 \mathrm{mg} / \mathrm{m}^{2}\right)$ with concurrent radiotherapy (on the first, twenty-second, and forty-third day, 5 times per week, mean dose $74 \mathrm{~Gy}$, range 70-78 Gy) at the Affiliated Cancer Hospital of Guangxi Medical University between January and December 2010 were included. After chemoradiotherapy, outcomes and long-term survival were evaluated. Immunohistochemistry was used to detect expression of ERCC1 protein in nasopharyngeal carcinoma. The relationship between the expression of ERCC1 and efficacy of concurrent chemoradiotherapy and long-term survival were analyzed. ERCC1 was expressed in $42.1 \%$ of cases. The expression of ERCC1 was correlated with $\mathrm{T}$
\end{abstract}


stage and clinical staging $(\mathrm{P}<0.05)$, but not with gender, age, or $\mathrm{N}$ stage. The response rate in the ERCC1-positive and ERCC1-negative groups was $75.0 \%$ and $97.7 \%$, respectively $(\mathrm{P}<0.05)$. In the 72 cases with follow-up available, 1-, 2-, and 3-year survival rates were 91.0, 83.3 , and $79.0 \%$; they were $92.4,87.8,80.5 \%$, respectively, in the ERCC1-positive group, and 87.9, 77.4, 77.4\%, respectively, in the ERCC1-negative group. The expression of ERCC1 may be a sensitive prognostic indicator of concurrent chemoradiotherapy in locally advanced nasopharyngeal carcinoma.

Key words: Nasopharyngeal carcinoma; Concurrent chemoradiotherapy; Excision repair cross-complementing gene 1 (ERCC1); Curative effect; Overall survival

\section{INTRODUCTION}

Nasopharyngeal cancer is a common malignancy for which radiotherapy is a commonly used treatment. In recent years, clinical studies have demonstrated that radiotherapy combined with chemotherapy (Ang et al., 2010), especially based on single-agent cisplatin (Segawa et al., 2010; Kreppel et al., 2012) is superior to radiotherapy alone. However, because platinum drugs usually target DNA, differences in the ability to repair DNA are often the direct cause for the observed differences in sensitivity to platinum drugs among patients (Kiyohara et al., 2006). DNA excision repair cross-complementing gene 1 (ERCC1) is a critical gene in the nucleotide excision repair pathway system that is located on chromosome 19q13.2-13.3 (Olaussen et al., 2007). Its normal expression is necessary to maintain normal function of the DNA repair system. At present, two methods are used to detect the expression of ERCC1, namely RT-PCR to detect ERCC1 mRNA levels and immunohistochemistry to detect ERCC1 protein. Of these two methods, the former is relatively expensive and requires a highly sophisticated technology, while the latter is more simple, inexpensive, and easy to use in routine clinical practice. Few studies have compared expression levels of ERCC1 protein by immunohistochemistry with the therapeutic effects of single-agent cisplatin chemotherapy with concurrent radiotherapy for locally advanced nasopharyngeal cancer. Thus, the present study has the aim of investigating potential correlations between expression of ERCC1 protein and the short- and long-term effects following single-agent cisplatin chemotherapy with concurrent radiotherapy.

\section{MATERIAL AND METHODS}

\section{Study population}

The study enrolled 76 patients (59 men and 17 women, age range 35-62 years, median 46 years) with undifferentiated non-keratinizing nasopharyngeal cancer hospitalized at our institution between January and December 2010. Of these, there was 1 patient in stage T1, 20 in T2, 33 in T3, and 22 in T4; 3 were in stage N0, 16 in N1, 43 in N2, and 14 in N3; there were 44 cases in stage III and 32 cases in stage IVA. 


\section{Inclusion criteria}

Inclusion criteria included the following: 1) diagnosis of locally advanced nasopharyngeal carcinoma non-differentiated non-keratinizing subtype by histopathology, and suitable for ERCC1 detection; 2) no previous radiotherapy or chemotherapy before biopsy; 3) receiving radiotherapy concurrent with single-agent chemotherapy with cisplatin; 4) performance status score (ECOG PS) $\leq 1$, and expected survival $\geq 3$ months; 5 ) at least one measurable tumor lesion on computed tomography/magnetic resonance imaging (MRI); 6) no obvious major organ dysfunction; 7) no history of other malignancies.

\section{Exclusion criteria}

Patients with the following were excluded: 1) distant metastasis before treatment; 2) severe medical illness; 3) double carcinoma; 4) had received prior chemotherapy.

\section{Treatment}

All patients were treated with concurrent chemoradiotherapy. For chemotherapy, 80 $\mathrm{mg} / \mathrm{m}^{2}$ of cisplatin was administered by $i v$ infusion; radiotherapy was delivered on the first, twenty-second, and forty-third day with a $6 \mathrm{MV}$ linear accelerator, conventional fractionated irradiation, 5 times per week, with an average dose of $74 \mathrm{~Gy}$ (range 70-78 Gy) in the nasopharyngeal area, $66 \mathrm{~Gy}$ in cervical lymph nodes, and $50 \mathrm{~Gy}$ in the supraclavicular field. Induction chemotherapy or adjuvant chemotherapy was not performed for any patient.

\section{Evaluation criteria}

RECIST 1.1 criteria and nasopharyngeal MRI were used to evaluate patients. Response rate (RR) was considered as complete response (CR) + partial response (PR), while the disease control rate (DCR) was considered as $\mathrm{CR}+\mathrm{PR}+$ stable disease.

\section{Follow-up}

Follow-ups were carried out through case query, review of imaging results of each reexamination, and telephone follow-up. By December 31, 2012, 4 cases were lost, and the dropout rate was 5.2\%. Progression-free survival (PFS) was defined as the time from the start of chemoradiotherapy to disease progression or death, while overall survival (OS) was defined as the time from the start of chemoradiotherapy to death or last follow-up.

\section{Immunohistochemical staining}

Fresh tissue was fixed in $10 \%$ formalin and cut into $4-\mu \mathrm{m}$ thick sections after embedding in paraffin. Sections were incubated at $60^{\circ} \mathrm{C}$ overnight, bathed in a xylene I, II, III cylinder for 10 min each time, then again washed in a graded ethanol series $(100,95,80,70,60 \%$ for $5 \mathrm{~min}$ each) for dewaxing. After dewaxing, sections were washed with running water before rinsing with PBS for three times for 3 min each. The CB method was used for antigen retrieval before rinsing three times in PBS for 3 min each. Sections were soaked in hydrogen peroxide for 15 
min, and then rinsed again with PBS three times for 3 min each. Primary antibody was added before incubation in a $37^{\circ} \mathrm{C}$ oven for $2 \mathrm{~h}$, following which samples were rinsed with PBS three times for 3 min each. Next, secondary antibody was added and samples were incubated in a $37^{\circ} \mathrm{C}$ oven for 30 min before rinsing with PBS three times, 3 min each. Freshly prepared diaminobenzidine solution was used for visualization, and slides were counterstained with hematoxylin after washing the sections with running water. Slides were quickly immersed in hydrochloric acid and alcohol after washing, and then washed before mounting and microscopic examination. PBS was used instead of primary antibody as a control. The double-blind method was used during examination of sections, i.e., sections were reviewed and scored independently by two pathologists who were unaware of the clinical data. To assess staining intensity, 400X fields were randomly selected from the upper, lower, left, and right parts of the sections, and staining intensity (no color for negative staining, light yellow for weak staining, brownish yellow for moderate staining, and brown for strong staining) in 5 fields was recorded. The percentage of positive cells $<25 \%$ was defined as negative expression (-), while expression in $>25 \%$ of cells was defined as positive expression (+). Positive expression was further sub-grouped into grade one/+ $(25-49 \%$ of cells), grade two/ $++(50-74 \%$ of cells $)$, and grade three/ $+++(\geq 75 \%$ of cells $)$.

\section{Statistical analysis}

Data were analyzed with the SPSS ver. 17.0 software. Differences between groups were compared with a $\chi^{2}$ test, non-parametric tests were analyzed with a Wilcoxon rank sum test, survival curves were drawn using the Kaplan-Meier method, and comparison of survival was performed using the log-rank method. A P $<0.05$ was considered to be statistically significant.

\section{RESULTS}

\section{Expression of ERCC1 protein in nasopharyngeal carcinoma}

ERCC1 protein was mainly distributed in the cytoplasm of tumor cells, observed as light yellow or brown yellow granular staining. Positive expression of ERCC1 was seen in $42.1 \%(32 / 76)$ of cases, among which 9 showed grade one/+, 12 cases showed grade two/++, and 11 cases showed grade three/+++ staining (Figure 1A-D).

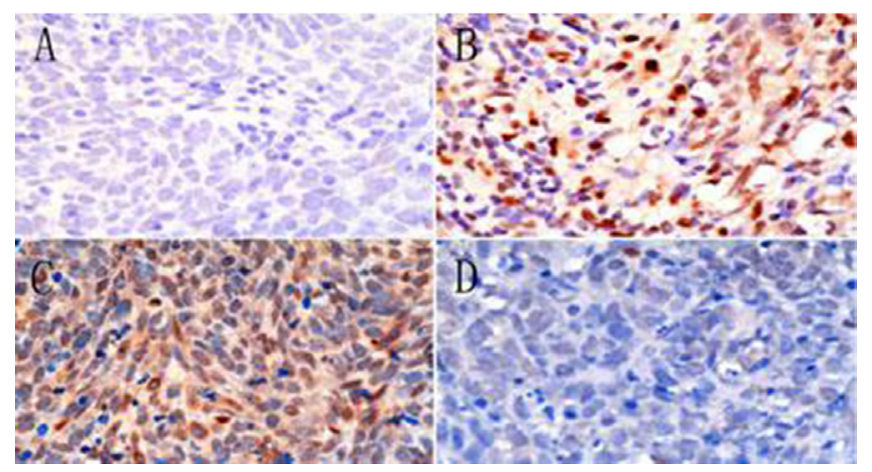

Figure 1. Expression of ERCC1 protein in nasopharyngeal carcinoma (SP, 400X). A. ERCC1 (-); B. ERCC1 (+); C. ERCC1 (++); D. ERCC1 (+++). 


\section{Association between ERCC1 expression and clinicopathological features in patients with nasopharyngeal carcinoma}

ERCC1 expression was not correlated with patient gender, age, or N stage $(\mathrm{P}>0.05)$, but was correlated with $\mathrm{T}$ stage and clinical staging $(\mathrm{P}<0.05)$. Moreover, the expression level of ERCC1 increased significantly with higher T stage or clinical staging (Table 1).

\begin{tabular}{|c|c|c|c|c|}
\hline \multirow[t]{2}{*}{ Clinicopathological parameter } & \multirow[t]{2}{*}{$\mathrm{N}$} & \multicolumn{2}{|c|}{ ERCC1 } & \multirow[t]{2}{*}{$P$} \\
\hline & & Positive & Negative & \\
\hline Gender & & & & 0.229 \\
\hline Male & 59 & $27(84.0)$ & $32(73.0)$ & \\
\hline Female & 17 & $5(16.0)$ & $12(27.0)$ & \\
\hline Age (years) & & & & 0.825 \\
\hline$<46$ & 37 & $14(43.8)$ & $23(52.3)$ & \\
\hline$>46$ & 39 & $18(56.2)$ & $21(47.7)$ & \\
\hline T stage & & & & 0.001 \\
\hline $\mathrm{T} 1{ }^{\circ}$ & 1 & $0(0)$ & $1(2.4)$ & \\
\hline T2 & 20 & $5(15.6)$ & $15(34.0)$ & \\
\hline T3 & 33 & $11(34.3)$ & $22(50.0)$ & \\
\hline $\mathrm{T} 4$ & 22 & $16(50.0)$ & $6(13.6)$ & \\
\hline $\mathrm{N}$ stage & & & & 0.805 \\
\hline No & 3 & $1(3.1)$ & $2(4.6)$ & \\
\hline N1 & 16 & $5(15.6)$ & $11(25.0)$ & \\
\hline N2 & 43 & $23(71.9)$ & $20(45.4)$ & \\
\hline N3 & 14 & $3(9.4)$ & $11(25.0)$ & \\
\hline Clinical staging & & & & 0.033 \\
\hline III & 44 & $14(43.8)$ & $30(68.2)$ & \\
\hline IVA & 32 & $18(56.2)$ & $14(31.8)$ & \\
\hline
\end{tabular}

\section{Short-term outcomes}

Short-term outcomes were evaluated in all 76 cases; 56 cases had a CR, 11 a PR, 3 with SD, and 6 with progression of disease. The RR was $88.16 \%$, while the DCR was $92.10 \%$.

\section{Relationship of ERCC1 expression with short-term outcomes}

In the ERCC1-positive cases, the RR was $75.0 \%$ (24/32), which was significantly different $(\mathrm{P}=0.008)$ from that observed in ERCC1-negative cases $[\mathrm{RR}=97.7 \%(43 / 44)]$. Moreover, among all ERCC1-positive patients, the RR was $88.9 \%(8 / 9)$ in patients with grade one expression, while in those with grade two and grade three expressions, the RR was $83.3 \%$ $(10 / 12)$ and $54.5 \%(6 / 11)$, respectively. Statistical analysis revealed that there was no difference in RR among patients with different grades of expression $(\mathrm{P}>0.05)$.

\section{Long-term outcomes}

Cumulative survival at 1,2, and 3 years was $91.0,83.3$, and $79.0 \%$ in the 72 patients with long-term follow-up. In ERCC1-negative patients, it was 92.4, 87.8, and 80.5\%, while it was 87.9, 77.4, and 77.4\% in ERCC1-positive patients, respectively. Furthermore, in ERCC1positive patients, OS decreased significantly with increasing grade of expression, i.e., OS in 
patients with grade one or two expression was significantly longer than that in patients with grade three expression $(\mathrm{P}<0.05$; Figure 2). No significant difference in OS was seen between ERCC1-positive and ERCC1-negative patients ( $\mathrm{P}>0.05$; Figure 3$)$.

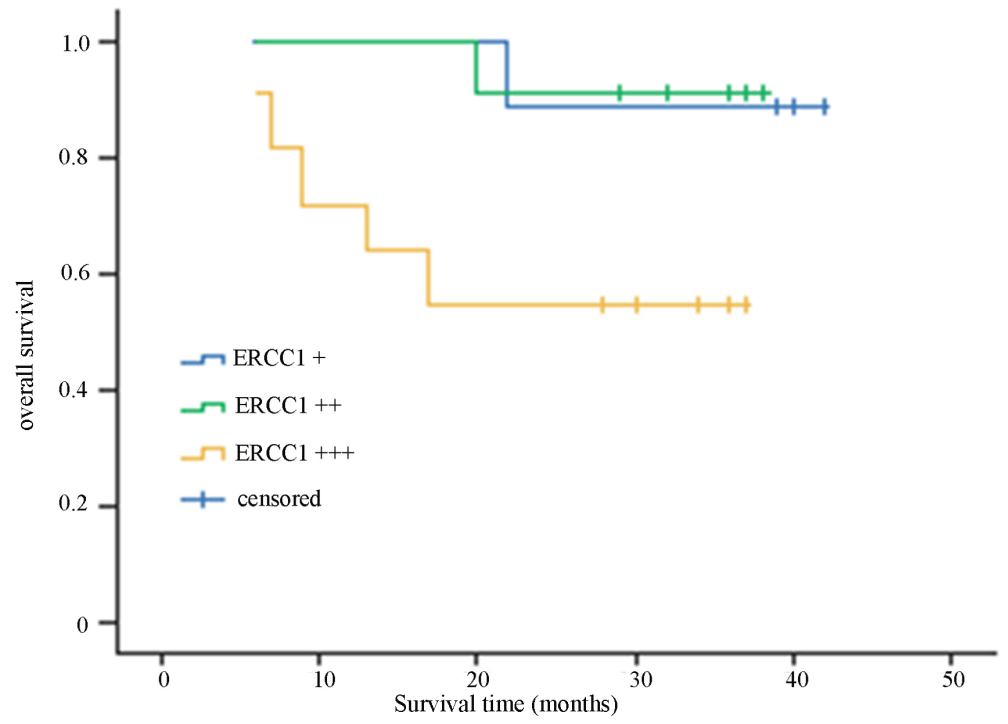

Figure 2. Overall survival of ERCC1-positive patients according to grade of staining.

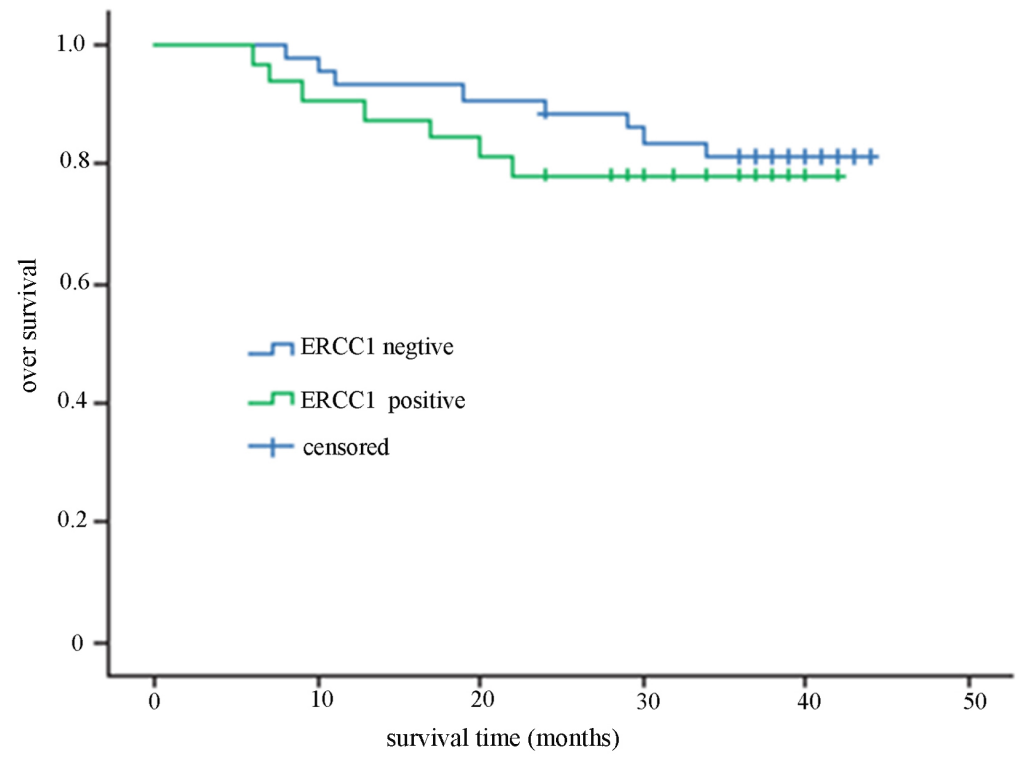

Figure 3. Overall survival of ERCC1-positive and ERCC1-negative patients. 


\section{DISCUSSION}

Tumors are the result of multiple changes in gene expression in normal cells, including changes in DNA repair genes, activation of tumor genes, and inactivation or deletion of tumor suppressor genes. Much attention has been placed on the DNA repair gene ERCC1. ERCC1 is not only closely related with the occurrence of multiple tumors, but it also is a predictor for the efficacy of therapy and prognosis of tumors. According to the literature (Cheng et al., 2002; Han et al., 2002), the expression of ERCC1 is low in nasopharyngeal carcinoma, indicating that there may be some correlation, and that low expression of ERCC1 may be a potential risk factor for nasopharyngeal carcinoma.

The results of our study showed that ERCC1 gene expression was seen in $42.1 \%$ (32/76) of cases. Moreover, ERCC1 expression was unrelated with patient gender, age, or N stage $(\mathrm{P}>0.05)$, but was correlated with $\mathrm{T}$ stage and clinical staging $(\mathrm{P}<0.05)$. Our results also showed that the expression level of ERCC1 increased significantly with higher clinical stages, and thus, ERCC1 may be a valid biological indicator to predict prognosis of nasopharyngeal carcinoma, although further research involving a larger number of samples is needed.

For patients with locally advanced nasopharyngeal carcinoma, concurrent chemoradiotherapy based on cisplatin is a standard treatment that has been demonstrated to prolong both PFS and OS. However, some patients do not appear to benefit from concurrent chemoradiotherapy, and thus, more individualized treatment options are needed. To date, a number of studies have reported correlation between ERCC1 expression and PFS and OS after platinumbased therapy in several cancers including colorectal, non-small cell lung, and ovarian tumors (Jiang et al., 2012; Li et al., 2013; Moxley et al., 2013). However, only a few studies have investigated such correlations in nasopharyngeal carcinoma, with inconsistent results. In the 76 patients who received concurrent chemotherapy and radiotherapy in the present study, total RR was 88.16 (67/76), while the RR in ERCC1-positive cases was 75.0\% (24/32) and 97.7\% $(43 / 44)$ in ERCC1-negative cases $(\mathrm{P}=0.008)$. Moreover, among all ERCC1-positive patients, there was no significant difference in short-term outcomes among the three grades of ERCC1 expression $(\mathrm{P}>0.05)$, implying that the predictive value of ERCC1 expression on short-term outcomes of concurrent chemoradiotherapy remains to be clarified.

There is still no definitive conclusion concerning the relationship between ERCC1 expression and survival of patients with nasopharyngeal carcinoma. Some studies have reported a negative correlation between the two, conjecturing that ERCC1 expression could be used to determine prognosis in these patients (Monzo et al., 2001; Lord et al., 2002). The results from our study showed that cumulative survival at 1, 2, and 3 years in 72 cases was $93.0 \%(67 / 72)$, $84.4 \%(61 / 72)$, and $72.2 \%(52 / 72)$, respectively. In addition, the long-term effect of concurrent chemoradiotherapy in patients with grade one or two expression was significantly different from that in those with grade three expression. This latter result is consistent with previous reports, further confirming that ERCC1 may be a predictor for prognosis of nasopharyngeal carcinoma (strong expression correlates with poor prognosis), although larger numbers of patients with follow-up are needed to validate this finding.

In conclusion, the expression of ERCC1 protein is closely related with short-term outcomes and long-term survival in patients with nasopharyngeal carcinoma following concurrent chemotherapy and radiotherapy. Detection of ERCC1 may help to assess the effects of concurrent chemoradiotherapy and to evaluate prognosis. Its potential clinical value thus warrants further research. 


\section{Conflicts of interest}

The authors declare no conflict of interest.

\section{ACKNOWLEDGMENTS}

Study on the combination of focused microwave hyperthermia with chemotherapy for patients with malignant hydrothorax and seroperitoneum (\#2010GXNSFA013243001).

\section{REFERENCES}

Ang KK, Harris J, Wheeler R, Weber R, et al. (2010). Human papillomavirus and survival of patients with oropharyngeal cancer. N. Engl. J. Med. 363:24-35.

Cheng L, Sturgis EM, Eicher SA, Spitz MR, et al. (2002). Expression of nucleotide excision repair genes and the risk for squamous cell carcinoma of the head and neck. Cancer 94:393-397.

Han WH, Li H, Xie L, Xu LG, et al. (2002). Plerosis of DNA array of normal human nasopharyngeal tissue and nasopharyngeal carcinoma. Zhonghuazhongliuzazhi 24:14-17.

Jiang J, Liang X, Zhou X, Huang R, et al. (2012). ERCC1 expression as a prognostic and predictive factor in patients with non-small cell lung cancer: a meta-analysis. Mol. Biol. Rep. 39:6933-6942.

Kiyohara C, Takayama K and Nakanishi Y (2006). Association of genetic polymorphisms in the base excision repair pathway with lung cancer risk: a meta-analysis. Lung Cancer 54:267-283.

Kreppel M, Danscheid S, Scheer M, Lüers JC, et al. (2012). Neoadjuvant chemoradiation in squamous cell carcinoma of the maxillary sinus: a 26-year experience. Chemother. Res. Pract. 2012:413589.

Li P, Fang YJ, Li F, Ou QJ, et al. (2013). ERCC1, defective mismatch repair status as predictive biomarkers of survival for stage III colon cancer patients receiving oxaliplatin-based adjuvant chemotherapy. Br. J. Cancer 108:1238-1244.

Lord RV, Brabender J, Gandara D, Alberola V, et al. (2002). Low ERCC1 expression correlates with prolonged survival after cisplatin plus gemcitabine chemotherapy in non-small cell lung cancer. Clin. Cancer Res. 8: 2286-2291.

Monzo M, Rosell R and Taron M (2001). Drug resistance in non-small cell lung cancer. Lung Cancer 34:91-94.

Moxley KM, Benbrook DM, Queimado L, Zuna RE, et al. (2013). The role of single nucleotide polymorphisms of the ERCC1 and MMS19 genes in predicting platinum-sensitivity, progression-free and overall survival in advanced epithelial ovarian cancer. Gynecol. Oncol. 130:377-382.

Olaussen KA, Mountzios G and Soria JC (2007). ERCC1 as a risk stratifier in platinum-based chemotherapy for nonsmallcell lung cancer. Curr. Opin. Pulm. Med. 13:284-289.

Segawa Y, Kiura K, Takigawa N, Kamei H, et al. (2010). Phase III trial comparing docetaxel and cisplatin combination chemotherapy with mitomycin, vindesine, and cisplatin combination chemotherapy with concurrent thoracic radiotherapy in locally advanced non-small-cell lung cancer: OLCSG 0007. J. Clin. Oncol. 28: 3299-3306. 